\section{Phospholipase D}

K. J. Lackner ${ }^{1}$ und D. Peetz ${ }^{2}$

${ }^{1}$ Institut für Klinische Chemie und Laboratoriumsmedizin, Universitätsmedizin Mainz, Mainz, Deutschland

${ }^{2}$ Institut für Labormedizin, Helios Klinikum Berlin-Buch,

Berlin, Deutschland

\section{Englischer Begriff phospholipase D}

Definition Enzym, das Phospholipide in Phosphatidsäure und das am Phosphat kovalent gebundene Molekül (Cholin, seltener Inositol u. a.) spaltet (s. Abbildung im Stichwort $\checkmark$ Phospholipase A2).
Beschreibung Phospholipase-D-Aktivität findet sich vorwiegend intrazellulär und ist dort u. a. in Signaltransduktion, Membrantransport und Zellzyklus involviert. Eine Reihe verschiedener Isoenzyme ist bekannt. Die Substratspezifität und Regulation dieser Isoenzyme unterscheidet sich z. T. erheblich.

\section{Literatur}

Exton JH (2002a) Phospholipase D-structure, regulation and function. Rev Physiol Biochem Pharmacol 144:1-94

Exton JH (2002b) Regulation of phospholipase D. FEBS Lett 531:58-61 